07

\title{
Механизм снижения прочности субмикроразмерных образцов ГЦК-металлов с нанокристаллической структурой
}

\author{
(C) Г.А. Малыгин \\ Физико-технический институт им. А.Ф. Иофрфе РАН, \\ Санкт-Петербург, Россия \\ E-mail: malygin.ga@mail.ioffe.ru
}

(Поступила в Редакцию 30 июня 2016 г.)

На основе дислокационно-кинетических уравнений и соотношений теоретически обсуждается эффект снижения прочности субмикроразмерных образцов металлов с нанокристаллической структурой при размерах поперечного сечения образцов $D<5 d$ по сравнению с прочностью образцов с $D \gg 5 d$, где $d$ - размер зерен. Ранее было установлено, что это снижение вызвано уходом части дислокаций через поверхность образца при работе однополюсных дислокационных источников в примыкающих к поверхности зернах. В работе показано, что поглощение решеточных дислокаций границами зерен и сопровождающее его зернограничное проскальзывание дополнительно снижают напряжение течения образцов — в равной степени, как тонких $(D<5 d)$, так и толстых $(D \gg 5 d)$.

DOI: $10.21883 /$ FTT.2017.02.44054.270

\section{1. Введение}

При пластической деформации кристаллических, в частности металлических, материалов существуют два размерных эффекта, влияющих на их прочность и пластичность. Один из них связан с поперечными размерами кристалла $D[1-3]$, другой — с наличием в деформируемом материале внутренних поверхностей: границ зерен и двойников, с плотностью соответственно $d^{-1}[3,4-7]$ и $\lambda^{-1}[3,8,9]$, где $d$ - размер зерен, $\lambda$ - ширина двойниковых ламелей. Влияние указанных размерных факторов оказывается особенно существенным, когда рассматриваемые размеры становятся порядка или меньше $1 \mu \mathrm{m}$. Согласно экспериментальным данным, предел текучести $\sigma_{y}$ нанокристаллов (nanopillars [1]) возрастает с уменьшением их поперечного размера $D$, как $\sigma_{y} \sim D^{-n}$, где $n=0.6-1.0$ [1-3]. Предел текучести нанокристаллического материала при величине зерен $d>10-30 \mathrm{~nm}$ подчиняется известному закону Холла-Петча (ХП) зернограничного упрочнения поликристаллов $\sigma_{y} \sim d^{-1 / 2}[4-6]$. Дальнейшее уменьшение размера зерен, однако, сопровождается снижением предела текучести (зернограничным разупрочнением) и возникновением обратного соотношения ХП $\sigma_{y} \sim d^{m}$, где согласно [5] $m=0.5$.

В литературе в настоящее время широко обсуждаются дислокационные механизмы, ответственные за размерные эффекты металлах с ГЦК-решеткой $(\mathrm{Cu}, \mathrm{Al}, \mathrm{Ni}$, $\mathrm{Au}$ и др.) [1-7]. Особый интерес и дискуссию вызывает в последнее время эффект взаимодействия размерных факторов $D$ и $d$, возникающий при деформации нанокристаллических (НК) образцов с поперечными размерами, сопоставимыми с размером зерен. Реальные и виртуальные (МД-моделирование) эксперименты с такими образцами показывают [10-12], что уменьшение их поперечного сечения приводит при $D<(3-5) d$ к существенному снижению у них предела текучести. Согласно [13] это снижение вызвано существенным ростом доли примыкающих к поверхности образца зерен, по сравнению с количеством зерен внутри образца. Зерна вблизи поверхности более слабо упрочняются из-за потери части дислокаций в результате их ухода через поверхность. Это приводит к общему снижению сопротивления образца пластической деформации. Результаты теоретического анализа этого механизма в рамках дислокационно-кинетического подхода [13], основанного на кинетических уравнениях и соотношениях для плотности дислокаций с учетом размерных факторов $D$ и $d$, показали их хорошее согласие с результатами экспериментов на НК-сплаве $\mathrm{Ni}-\mathrm{W}[10,11]$ и МК Ag [14].

Дополнительным механизмом разупрочнения нанокристаллических образцов с поперечными размерами $D<5 d$ может быть обсуждаемый в [12] механизм разупрочнения НК материала в результате проскальзывания (sliding) примыкающих к поверхности образца зерен по их границам. На существование зернограничного проскальзывания указывает возникновение ступенек на границах приповерхностных нанозерен при сжатии образцов НК $\mathrm{Pt}$ с поперечными размерами $D=5 d$ при комнатной температуре, а также результаты молекулярно-динамического моделирования деформации НК-платины [12]. Согласно [5,15] проскальзывание по границам зерен в процессе деформации является результатом поглощения ими решеточных дислокаций, поскольку собственных зернограничных дислокаций для этого недостаточно.

Таким образом, при поперечных размерах НК-образцов, сопоставимых с размерами нанозерен, имеются два механизма снижения деформационного упрочнения приповерхностных зерен. Первый - в результате роста относительной доли слабо упрочняющихся зерен вблизи поверхности образца из-за ухода части дислокаций из 
них через поверхность (аннигиляцию с ней). Второй в результате поглощения решеточных дислокаций границами зерен (аннигиляции их в границах). Первый механизм в рамках дислокационно-кинетического подхода был рассмотрен в [13]. Целью настоящей работы является анализ второго механизма в рамках аналогичного подхода.

\section{2. Основные уравнения и соотношения}

Кинетическое уравнение для плотности дислокаций $\rho$, содержащее в своей правой части характерные для нанокристаллических материалов и микро- и наноразмерных образцов кинетические процессы, имеет вид

$$
\frac{d \rho}{d t}=\frac{\beta}{d} u \rho-\frac{\rho}{t_{g b}}-h_{a} u \rho^{2},
$$

где $t$ - время, $u$ - скорость дислокаций,

$$
\beta(d, D)=1-\frac{\Delta S}{S}=(1-d / D)^{2}, \quad D>d,
$$

$\Delta S$ - суммарная площадь приповерхностных зерен в поперечном сечении круглого образца, содержащих однополюсные источники Франка-Рида, $S$ - площадь сечения, $d-$ размер зерен, $D-$ поперечный размер образца [13]. Первое слагаемое в правой части уравнения (1a) описывает скорость роста плотности дислокаций в поликристалле из-за наличия границ зерен как барьеров для дислокаций. Второе слагаемое - скорость аннигиляции решеточных дислокаций в границах зерен, где $t_{g b}$ - характерное время аннигиляции $[5,15]$,

$$
t_{g b}=d^{2} / 4 \eta_{g b} D_{g b},
$$

$D_{g b}-$ коэффициент зернограничной диффузии, $\eta_{g b} \approx \mu b^{3} / k_{\mathrm{B}} T, \quad \mu-$ модуль сдвига, $b$ - вектор Бюргерса, $k_{\mathrm{B}}$ - постоянная Больцмана, $T$ - температура. Третий член - скорость аннигиляции винтовых участков дислокационных петель поперечным скольжением, где $h_{a}$ - характерное расстояние их аннигиляции. Уравнение (1a) отличается от аналогичного уравнения в [13] наличием в правой его части дополнительного слагаемого $-\rho / t_{g b}$.

В условиях деформации с постоянной скоростью $\dot{\varepsilon}=m^{-1} \dot{\gamma}$ для скорости изменения плотности дислокаций со временем $t$ имеет место соотношение $d \rho / d t=(d \rho / d \gamma) \dot{\gamma}$, где $\dot{\gamma}=b \rho u-$ скорость сдвиговой деформации. Подставляя его в (1a), получаем уравнение

$$
\begin{gathered}
\frac{d \rho}{d \gamma}=\frac{\beta(d, D)}{b d}-\left(k_{a}+k_{g b}(d)\right) \rho \\
k_{g b}(d)=\eta_{g b} \frac{4 D_{g b}}{m \dot{\varepsilon} d^{2}}=\left(\frac{d_{g b}}{d}\right)^{2}, \quad d_{g b}=\left(\frac{4 \eta_{b} D_{g b}}{m \dot{\varepsilon}}\right)^{1 / 2},
\end{gathered}
$$

где $k_{a}=h_{a} / b-$ коэффициент аннигиляции винтовых дислокаций, $k_{g b}$ - коэффициент аннигиляции краевых участков дислокационных петель в границах зерен, $m=m_{\mathrm{T}}-$ фактор Тейлора для поликристалла. При выводе соотношений $(2 \mathrm{~b})$ было принято во внимание, что скорость дислокаций $u$ равна $m_{\mathrm{T}} \dot{\varepsilon} / b \rho$. Интегрирование уравнения (2a) позволяет найти зависимость плотности дислокаций от деформации $\gamma=m_{\mathrm{T}} \varepsilon$ и размерных факторов $d$ и $D$,

$$
\begin{aligned}
\rho(\varepsilon, d, D)= & \left(\frac{\beta(d, D)}{\left(k_{a}+k_{g b}(d)\right) b d}\right) \\
& \times\left[1-\exp \left(-m_{\mathrm{T}}\left(k_{a}+k_{g b}(d)\right) \varepsilon\right],\right.
\end{aligned}
$$

а также, согласно уравнению Тейлора для деформационного (дислокационного) упрочнения $\sigma=m \alpha \mu b \rho^{1 / 2}$, найти зависимость напряжения течения от этих факторов

$$
\begin{aligned}
\sigma(\varepsilon, d, D)= & m_{\mathrm{T}} \alpha \mu=\left(\frac{\beta(d, D) b}{\left(k_{a}+k_{g b}(d)\right) d}\right)^{1 / 2} \\
& \times\left[1-\exp \left(-m_{\mathrm{T}}\left(k_{a}+k_{g b}(d)\right) \varepsilon\right],\right.
\end{aligned}
$$

где $\alpha-$ коэффициент взаимодействия дислокаций, $k_{g b}(d)=\left(d_{g b} / d\right)^{2}$.

\section{3. Влияние размерных факторов на напряжение течения}

На рис. 1 кривые демонстрируют в координатах $\sigma / \mu-D$ зависимость согласно уравнению (4) напряжения течения $(\varepsilon=0.2 \%)$ от поперечного размера $D$

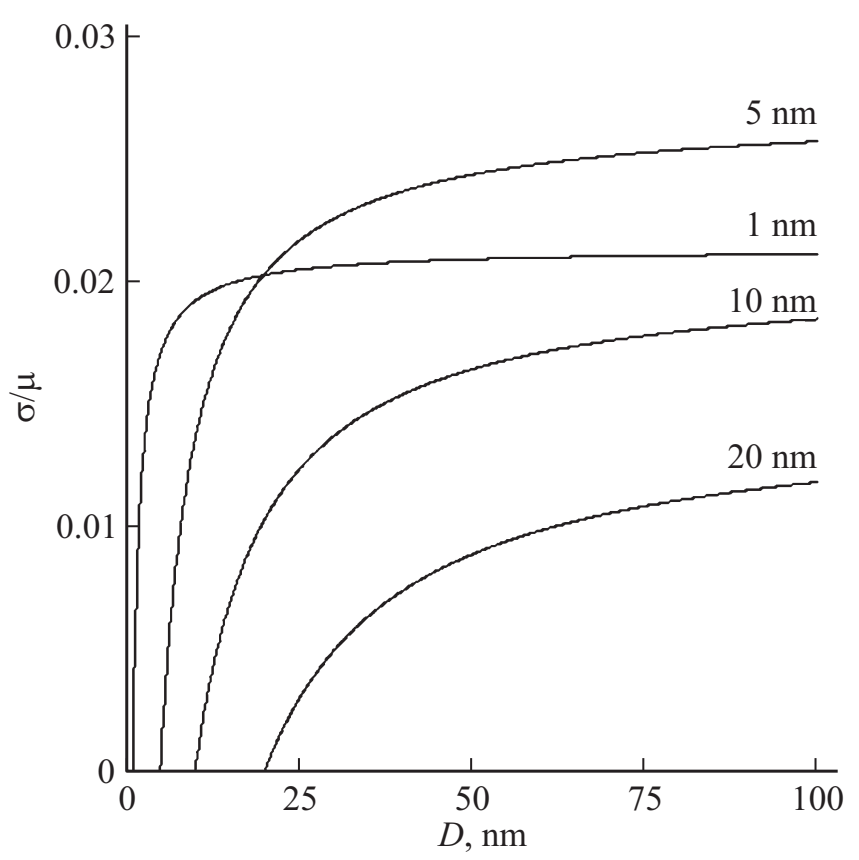

Рис. 1. Зависимость напряжения течения при деформации $0.2 \%$ от размера поперечного сечения НК-образца $D$ в координатах $\sigma / \mu-D$ согласно уравнению (4). Цифры у кривых величина зерен в $\mathrm{nm}$. 


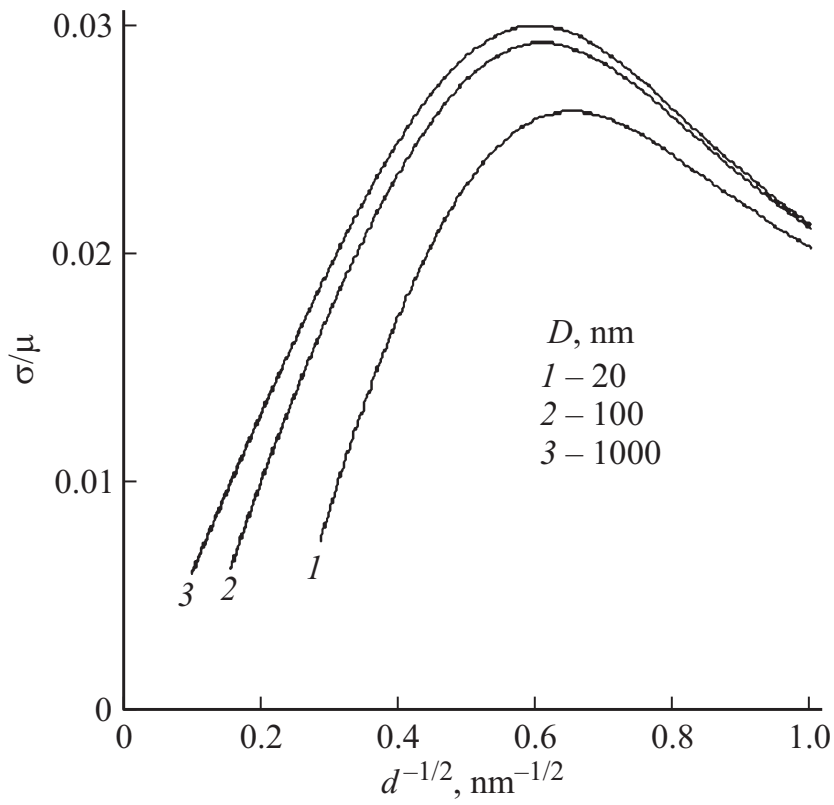

Рис. 2. Зависимость напряжения течения при деформации $0.2 \%$ от размера зерен $d$ в координатах Холла-Петче $\sigma / \mu-d^{-1 / 2}$ согласно уравнению (4) при трех размерах сечения НК-образцов.

образцов с разной величиной зерна при значениях параметров $\alpha=0.5, b=0.28 \mathrm{~nm}, k_{a}=2$ и $d_{g b}=40 \mathrm{~nm}$. Видно, что при приближении поперечных размеров образцов к размеру зерен напряжения течения существенно снижаются в сравнении с напряжениями течения образцов с поперечными размерами $D \gg d$. Напряжение течения таких образцов непрерывно возрастает с уменьшением размера зерен в соответствии с законом ХП; и только при $d<5 \mathrm{~nm}$ оно снижается из-за аннигиляции дислокаций в границах зерен. Это снижение наглядно иллюстрируют зависимости напряжений течения $(\varepsilon=0.2 \%)$ от размера зерен в координатах ХП $\sigma / \mu-d^{-1 / 2}$ (рис. 2) и разной величине поперечных размеров образцов $D$. Видно, что чем тоньше образец, тем меньше его сопротивление пластической деформации. Это сопротивление дополнительно снижается в области сверхмалых размеров нанозерен в результате зернограничного разупрочнения нанокристаллического материала.

На рис. 3 приведены экспериментальные данные [12] по зависимости напряжений течения $(\varepsilon=0.2 \%)$ образцов нанокристаллической Pt с размером зерен $d=12 \mathrm{~nm}$ от поперечного сечения образцов $D$ при изменении его в широком диапазоне. Из этих данных видно, что напряжение течения практически не зависит от размера сечения при $D>10 d \mathrm{~nm}$, а при $D=5 d=60 \mathrm{~nm} \mathrm{оно}$ существенно снижается. Кривая 1 на рис. 3 иллюстрирует результат расчета зависимости напряжений течения НК-платины согласно уравнению (4) от размера сечения $D$ при $d=12 \mathrm{~nm}, d_{g b}=40 \mathrm{~nm}, \varepsilon=0.2 \%$ и $\mu=68 \mathrm{GPa}$, значения остальных параметров приведены выше. Кривая 2 на этом рисунке - расчет напряжения течения согласно уравнению (4) в отсутствие поглощения дислокаций границами нанозерен $\left(d_{g b}=0\right)$. Незначительная разница напряжений между кривыми 1 и 2 связана с малой величиной деформации $(0.2 \%)$, и, следовательно, с малой плотностью дислокаций в зернах. С ростом деформации влияние аннигиляции в границах на напряжение течения становится более существенным. На рис. 3 кривые 3 и 4 иллюстрируют это обстоятельство при величине деформации $\varepsilon=1 \%$. Согласно уравнению (4) отношение напряжений течений в отсутствие и при наличии аннигиляции дислокаций в границах не зависит от размера сечения, в том числе и при размере сечения $60 \mathrm{~nm}$. Из приведенных на рис. 3 данных видно также, что экспериментальное значение напряжения течения образца с величиной зерна $d=60 \mathrm{~nm}$ на $20 \%$ меньше расчетного. Неясно, чем вызвана эта разница, находится ли она в пределах разброса экспериментальных точек или, как это предполагается в [12], связана со специфическим вкладом зернограничного проскальзывания в напряжение течения.

На напряжение течения влияют не только размерные факторы, но и величина параметра $d_{g b}=\left(4 \eta_{b} D_{g b} / m_{\mathrm{T}} \dot{\varepsilon}\right)^{1 / 2}$, зависящая от скорости деформации и коэффициента зернограничной диффузии $D_{g b}=D_{g b}^{0} \exp \left(-U_{g b} / k_{\mathrm{B}} T\right)$, где $U_{g b}$ - энергия активации зернограничной диффузии, $D_{g b}^{0} \approx 10^{-5} \mathrm{~m}^{2} / \mathrm{s}$ - предэкспоненциальный множитель.

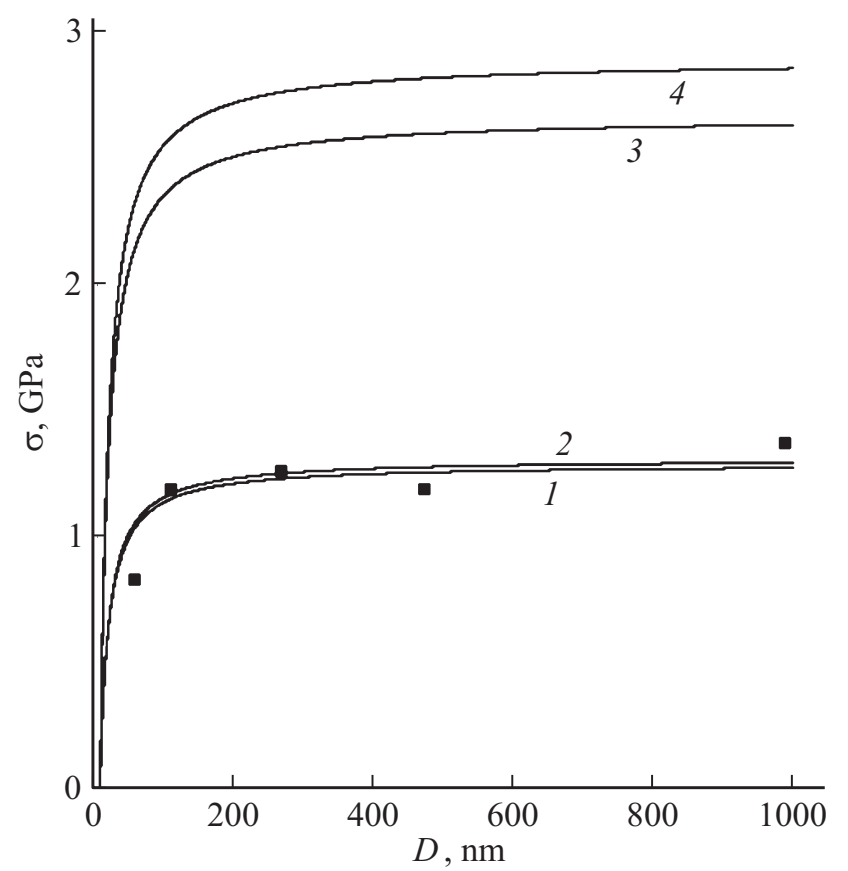

Рис. 3. Зависимость напряжения течения при деформации $0.2 \%$ (кривые 1 и 2) и $1.0 \%$ (кривые 3 и 4 ) образцов НК Pt $(d=12 \mathrm{~nm})$ от поперечного сечения образцов $D$ согласно уравнению (4) в присутствии (кривые 1 и 3) и в отсутствие (кривые 2 и 4) поглощения дислокаций границами зерен. Экспериментальные точки - данные [12]. 
Чем выше температура и ниже скорость деформации, тем больше величина этого параметра и больше коэффициент аннигиляции дислокаций $k_{g b}$ и меньше напряжение течения. Интересно оценить величину энергии активации зернограничной диффузии $U_{g b}$ в $\mathrm{Pt}$, контролирующую аннигиляцию дислокаций в границах зерен, и, согласно данным [12], образование ступенек на границах зерен, выходящих на поверхность образца. Для оценки энергии активации имеем соотношение

$$
U_{g b}=k_{\mathrm{B}} T \ln \left(\frac{4 \eta_{b} D_{g b}^{0}}{m_{\mathrm{T}} \dot{\varepsilon} d_{g b}^{2}}\right),
$$

где $\eta_{b} \approx 550$. Подставляя в (5) приведенные выше значения параметров, получаем, что при $T=293 \mathrm{~K}$ и скорости деформации $\dot{\varepsilon}=10^{-3} \mathrm{~s}^{-1}$ процесс аннигиляции дислокаций в зернах и проскальзывание по границам может обеспечить величина энергии зернограничной самодиффузии $U_{g b} \approx 0.9 \mathrm{eV}$. Она равна примерно $1 / 3$ энергии активации объемной самодиффузии платины $U_{S D}=2.9 \mathrm{eV}$. Приблизительно такое же соотношение между энергиями зернограничной, $U_{g b} \approx 0.71 \mathrm{eV}$, и объемной, $U_{S D}=2.8 \mathrm{eV}$, самодиффузии в $\mathrm{Ni}$ было найдено в [16] при исследовании ползучести образцов нанокристаллического сплава $\mathrm{Ni}-\mathrm{P}$ с размером зерен $28 \mathrm{~nm}$.

\section{4. Зернограничное проскальзывание}

При обсуждении влияния проскальзывания по границам зерен на разупрочнение образцов с поперечными размерами, сопоставимыми с размерами нанозерен, часто предполагают, что зернограничное проскальзывание является автономным механизмом деформации, вызывающим концентрацию напряжений в тройных стыках зерен и эмиссию из них дислокаций. Аргументом в пользу автономности служит отсутствие дислокаций внутри нанозерен после разгрузки образца. Но при МД-моделировании деформации НК-платины $(d=12 \mathrm{~nm})$ видно, например, что дислокации испускаются границами и стыками зерен, а после пересечения тела зерна поглощаются границами [12]. При $d=12 \mathrm{~nm}$ и длине дислокационной петли $4 d$, расширяющейся внутри кубического зерна объемом $d^{3}$, динамическая плотность дислокаций в зерне, обеспечивающая его деформацию $\varepsilon=b / d=2.3 \%$, где $b=0.28 \mathrm{~nm}$, составляет $\rho=4 / d^{2} \approx 1.5 \cdot 10^{16} \mathrm{~m}^{-2}$. Именно такого порядка плотность дислокаций была обнаружена в НК-никеле $(d=24 \mathrm{~nm})$ [17]. Но только после деформации при $77 \mathrm{~K}$. После деформации при комнатной температуре дислокации внутри нанозерен отсутствовали, поскольку были поглощены границами зерен.

Согласно [5,15] зернограничное проскальзывание является результатом перехода решеточных дислокаций в границы зерен. Без внешних (extrinsic) по отношению к границам дислокаций собственных (intrinsic) зернограничных дислокаций недостаточно для осуществления этого проскальзывания. Согласно этой точке зрения проскальзывание является вторичным процессом. Первичным является поглощение решеточных дислокаций границами, что снижает плотность дислокаций в объеме нанозерен и уменьшает тем самым их деформационное упрочнение. В отсутствие такого поглощения дислокации накапливаются внутри нанозерен, как это было продемонстрировано в [17], что сопровождается ростом деформационного упрочнения зерен вследствие взаимодействия дислокаций на некомпланарных плоскостях скольжения друг с другом как с дислокациями леса, что также было зафиксировано в [17].

При $k_{g b}(d)=\left(d_{g b} / d\right)^{2}$ и $d_{g b}=0$, т. е. в отсутствие аннигиляции решеточных дислокаций в границах зерен, зависимость плотности дислокаций внутри зерен от деформации и размерных факторов имеет согласно (3) вид

$$
\rho_{0}(\varepsilon)=\left(\frac{\beta(d, D)}{k_{a} b d}\right)\left[1-\exp \left(-m_{\mathrm{T}} k_{a} \varepsilon\right)\right] .
$$

При поглощении части дислокаций (6a) границами зерен изменение плотности дислокаций внутри зерен $\rho_{g}(\varepsilon)$ и поглощенных границами $\rho_{b}(\varepsilon)$ с деформацией определяют уравнения

$$
\begin{gathered}
\rho_{g}(\varepsilon)=\left(\frac{\beta(d, D)}{\left(k_{a}+k_{g b}(d)\right) b d}\right)\left[1-\exp \left(-m_{\mathrm{T}}\left(k_{a}+k_{g b}(d)\right) \varepsilon\right)\right], \\
\rho_{b}(\varepsilon)=\rho_{0}(\varepsilon)-\rho_{g}(\varepsilon),
\end{gathered}
$$

или в относительных долях $N_{g}$ - и $N_{b}$-соотношения

$$
\begin{gathered}
N_{g}(\varepsilon)=\frac{\rho_{g}(\varepsilon)}{\rho_{0}(\varepsilon)} \\
=\left(\frac{k_{a}}{k_{a}+k_{g b}(d)}\right) \frac{1-\exp \left(-m_{\mathrm{T}}\left(k_{a}+k_{g b}(d)\right) \varepsilon\right)}{1-\exp \left(-m_{\mathrm{T}} k_{a} \varepsilon\right)} \\
N_{b}(\varepsilon)=\frac{\rho_{b}(\varepsilon)}{\rho_{0}(\varepsilon)}=1-N_{g}(\varepsilon)
\end{gathered}
$$

На рис. 4, а кривые демонстрируют согласно соотношениям (7) зависимости соответствующих долей от деформации $\varepsilon$ при $d=12 \mathrm{~nm}, d_{g b}=40 \mathrm{~nm}$ и $D=120 \mathrm{~nm}$. Видно, что при указанном значении параметра $d_{g b}$ доля дислокаций, поглощенных границами зерен в начале процесса их аннигиляции, существенно меньше доли дислокаций в объеме зерен. Но в конце этого процесса она в 5 раз превышает долю дислокаций внутри зерен, и это соотношение не зависит от поперечного размера образцов. При вдвое меньшем значении параметра $d_{g b}=20 \mathrm{~nm}$, т.е. при снижении температуры или росте скорости деформации, соотношение между этими долями в конце процесса аннигиляции оказывается близким к единице (рис. 4,b). При дальнейшем снижении температуры или увеличении скорости деформации $\left(d_{g b}=10\right)$ практически все дислокации оказываются сконцентрированными внутри зерен. 

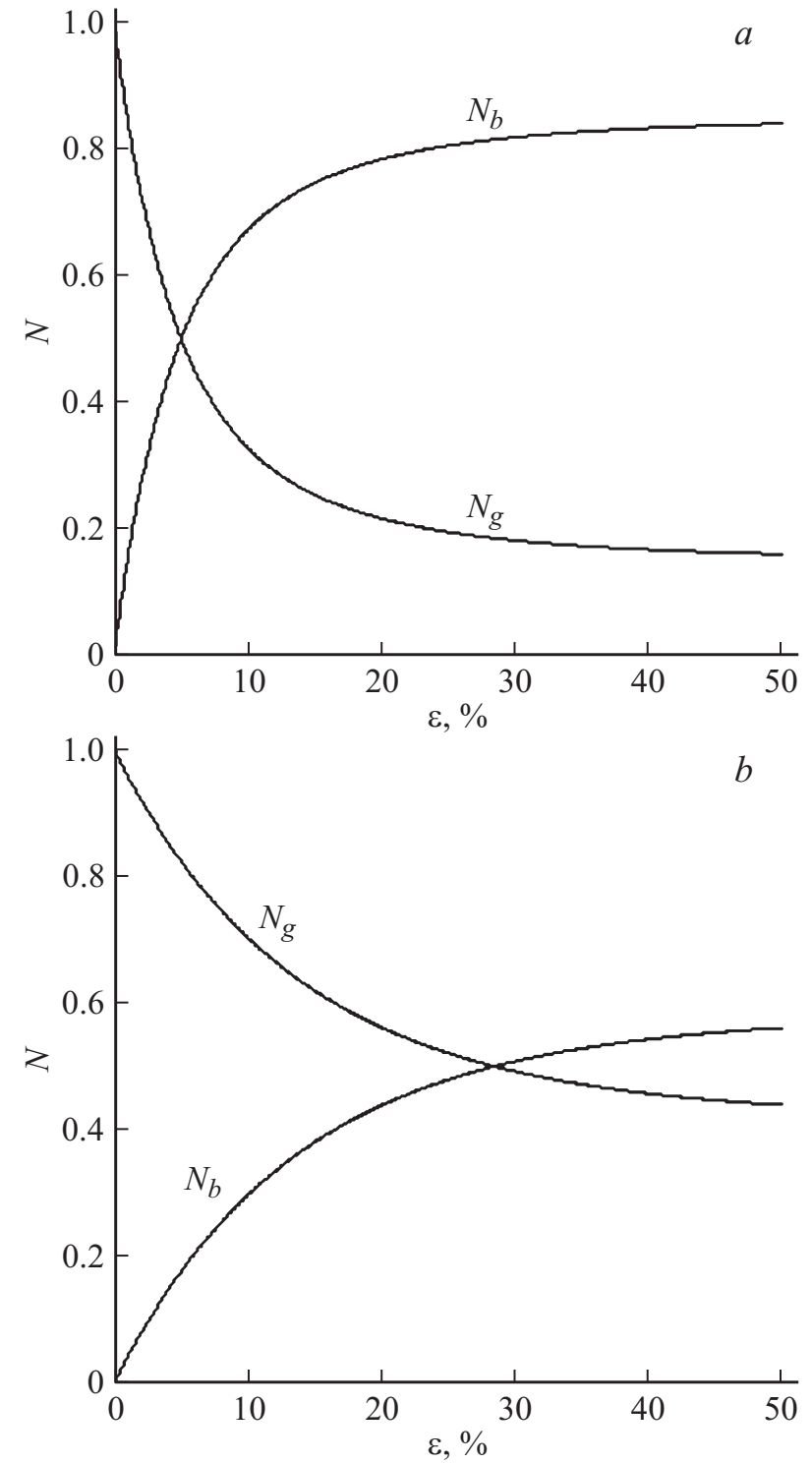

Рис. 4. Зависимость относительных долей плотности дислокаций (6a), поглощенных $\left(N_{b}\right)$ и не поглощенных $\left(N_{g}\right)$ границами зерен, от деформации $\varepsilon$ при величине параметра $d_{g b}=40 \mathrm{~nm}(a)$ и $d=20 \mathrm{~nm}(b)$ и $d=12 \mathrm{~nm}, D=120 \mathrm{~nm}$.

Поглощение дислокаций границами зерен инициирует деформацию пластического сдвига соседних зерен относительно друг друга, $\gamma_{b}(\varepsilon)=b \rho_{b b}(\varepsilon) \lambda_{b}$, где $\lambda_{b}=d-$ расстояние свободного пробега дислокаций в границе, $\rho_{b b}=\left(d / 6 \delta_{b}\right) \rho_{b}-$ плотность поглощенных границами дислокаций в границах кубического по форме зерна, $\delta_{b}=2 b$ - ширина границ. На рис. 5, $a$ приведены результаты расчета сдвиговых деформаций в образцах НК Pt в границах $\gamma_{b}$ и внутри зерен, $\gamma_{g}(\varepsilon)=b \rho_{g}(\varepsilon) d$, при $d=12 \mathrm{~nm}, D=120 \mathrm{~nm}$ и $d_{g b}=40 \mathrm{~nm}$. Видно, что локализованный в границе сдвиг $\gamma_{b}$ существенно больше распределенного по объему зерна сдвига $\gamma_{g}$. На поверхность образца при кубической форме зерна могут выходить 4 границы. Поскольку они расположены друг от друга на расстоянии $d$, а их плоскости составляют с осью кристалла в среднем угол $\approx 45^{\circ}$, распределенная равномерно по высоте образца деформация зернограничного сдвига $\gamma_{a v}=(4 / \sqrt{2})(b / d) \gamma_{b} \approx 0.066 \gamma_{b}$ оказывается значительно меньше равномерно распределенной по объему зерен сдвиговой деформации $\gamma_{g}$, особенно, при деформациях образца $\varepsilon<10 \%$. Это обстоятельство иллюстрирует кривая $\gamma_{a v}(\varepsilon)$ на рис. 5, $a$.

В местах выхода границ зерен на поверхность образца на ней образуются ступеньки шириной $w_{b}=\gamma_{b} d$ и высотой $h_{b}=\gamma_{b} d / \sqrt{2}$. В отличие от границ зерен ступеньки $h_{g}=\gamma_{g} d / \sqrt{2}$ равномерно распределены по объему примыкающих к поверхности образца зерен. На рис. 5, $b$ приведены результаты расчета величины ступенек $h_{b}$ и $h_{g}$ в НК-платине.
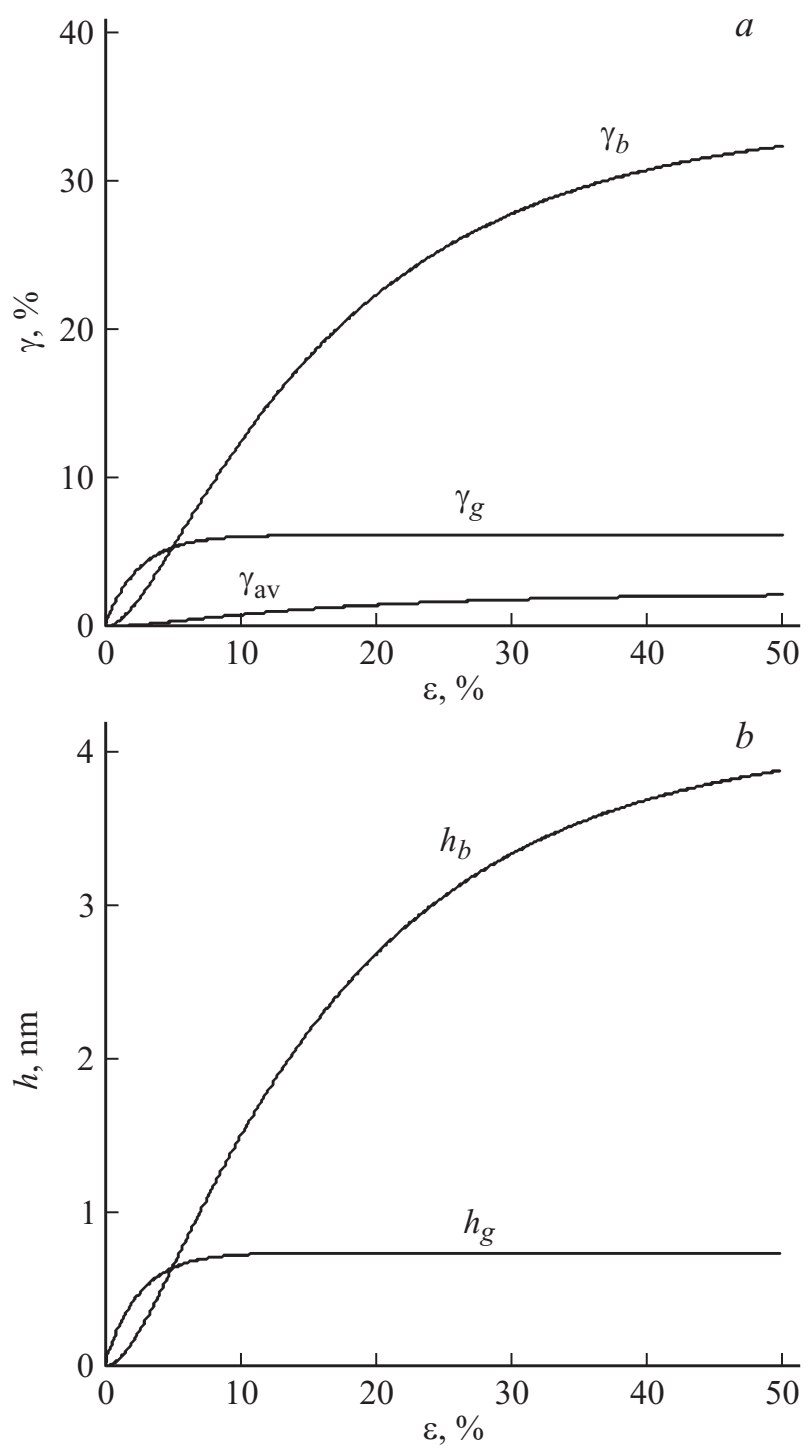

Рис. 5. Зависимость деформации сдвига по границам $\gamma_{b}$ и в объеме $\left(\gamma_{g}\right)$ зерен, и усредненной по образцу зернограничной сдвиговой деформации $\gamma_{a v}(a)$ и $(b)$ величины ступенек на границах $h_{b}$ и распределенных по объему $\left(h_{g}\right)$ зерен от полной деформации НК-образца $\varepsilon$. 


\section{5. Заключение}

Результаты анализа деформационного поведения субмикроразмерных образцов НК-металлов с ГЦК-решеткой на основе дислокационно-кинетических уравнений и соотношений показывают, что поглощение решеточных дислокаций границами зерен, вызывающее зернограничное проскальзывание, сопровождается снижением напряжений течения НК-материала. Это снижение не зависит от размера поперечного сечения нанокристаллических образцов, т.е. в равной степени относится и к „тонким“, и к „толстым“ образцам. Наблюдаемый в эксперименте $D$-размерный эффект связан, как и в отсутствие проскальзывания по границам зерен, с уходом части дислокаций через поверхность образца при работе вблизи нее однополюсных дислокационных источников.

\section{Список литературы}

[1] M.D. Uchic, P.A. Shade, D.M. Dimiduk. Ann. Rev. Mater. Res. 39, 361 (2009).

[2] J.R. Greer, J.T.M. de Hosson. Progr. Mater. Sci. 56, 654 (2011).

[3] Г.А. Малыгин. УФН 181, 1129 (2011).

[4] M.A. Meyers, A. Mishra, D.J. Benson. Progr. Mater. Sci. 51, 427 (2006).

[5] Г.А. Малыгин. ФТТ 49, 961 (2007).

[6] R.W. Armstrong. In: Mechanical Properties of Nanocrystalline Materials. Ch. 3. / Ed. J.C.M. Li. World Sci., N. Y. Publ. (2009). P. 1-34.

[7] Р.А. Андриевский, А.М. Глезер. УФН 179, 337 (2009).

[8] L. Lu, X. Chen, X. Huang, K. Lu. Science 323, 607 (2009).

[9] Г.А. Малыгин. ФТТ 53, 711 (2011).

[10] D. Jang, J.R. Greer. Scripta Mater. 64, 77 (2011).

[11] D. Jang, C. Cai, J.R. Greer. NanoLett. 11, 1743 (2011).

[12] X.W. Gu, C.N. Loynachan, Zh. Wu, Y.-W. Zhang, D.J. Srolovitz, J.R. Greer. NanoLett. 12, 6385 (2012).

[13] Г.А. Малыгин. ФТТ 54, 523 (2012).

[14] X. Chen, A. Ngan. Scripta Mater. 64, 717 (2011).

[15] Г.А. Малыгин. ФТТ 37, 2281 (1995).

[16] D.I. Wang, Q.P. Kong, J.P. Shui. Scripta Metal. Mater. 31, 47 (1994).

[17] X-L. Wu, E. Ma. Appl.Phys. Lett. 88, 231911 (2006). 